\title{
When the Labs Closed: Graduate Students' and Postdoctoral Fellows' Experiences of Disrupted Research During the COVID-19 Pandemic
}

Celeste Suart

McMaster University https://orcid.org/0000-0003-1949-9377

Theresa Nowlan Suart

Queen's University https://orcid.org/0000-0001-8329-4400

Katherine Graham

McMaster University https://orcid.org/0000-0001-6434-3393

Ray Truant ( $\nabla$ truantr@mcmaster.ca )

McMaster University https://orcid.org/0000-0003-2542-6641

\section{Research Article}

Keywords: COVID-19, graduate studies, lockdown, higher education policy, post doctoral fellows

Posted Date: August 17th, 2020

DOI: https://doi.org/10.21203/rs.3.rs-59050/v1

License: (c) (i) This work is licensed under a Creative Commons Attribution 4.0 International License. Read Full License

Version of Record: A version of this preprint was published at FACETS on June 22nd, 2021. See the published version at https://doi.org/10.1139/facets-2020-0077. 


\section{Abstract}

Physical distancing measures in response to the COVID-19 pandemic resulted in widespread laboratory closures. This study aimed to examine the impact of this disruption on graduate students and postdoctoral fellows completing laboratory-based research in Canada. We used an anonymous online survey and semi-structured interviews to document the experiences of graduate students and postdoctoral fellows during laboratory closures and following the transition to working from home. We employed mixed-method analysis of survey and interview data to identify shared experiences, feelings, concerns, and supports.

Respondents' emotions reported from different points during the COVID-19 laboratory closures align with previously described Kübler-Ross model of grief following change. Respondents describe closure processes as chaotic and confusing, primarily due to inconsistent communication between graduate students, postdoctoral fellows, supervisors, and administration. Respondents reported increased anxiety during closures, however, those who experienced uniform laboratory closures also developed a sense of solidarity and safety while those with staggered shutdowns did not. Graduate students and postdoctoral fellows reported increased indications of distress while working from home. Four key barriers to working from home were described; technical issues, domestic distractions, decreased motivation, and poor mental health. Five key supports while working at home were also identified, including financial security, social connections, establishing and maintaining routines, mental health support, and support from supervisors and administration. Respondents had concerns of how COVID-19 might impact them, including decreasing competitiveness applicants while limiting future employment opportunities. Finally, we outline five types of supports which can be implemented by supervisors and administrators to support graduate students and postdoctoral fellows to return to the laboratory, including; personal protective equipment and protocols, understanding and empathy, guidance and direction, timeline support, and financial support. Overall, we document shared experiences of respondents during the COVID-19 laboratory shutdown and identify areas of improvement in the event widespread laboratory closures occur in the future.

\section{Introduction}

In spring 2020, researchers worldwide experienced an unprecedented wave of laboratory closures in response to the COVID-19 pandemic. In mere weeks, nearly all research was put on pause to prevent further spread of SARS-CoV-2 [1].

Widespread closure of laboratories was unprecedented. Research laboratories have had emergency shutdowns following natural disasters before, such as hurricanes, floods, and earthquakes [2-5]. However, these shutdowns differ from the COVID-19 laboratory shutdown on two key factors. First, most natural disasters are geographically contained, as opposed to impacting a worldwide population. Second, 
although catastrophic, natural disasters occur over a finite amount of time. Following a hurricane or earthquake, researchers can begin to make plans for what is necessary to reopen. Closures due to COVID19 did not come with this timescale, with many not knowing when they will be able to return to pre-COVID level operating capacity.

Researchers and institutions had to react quickly to close laboratories, by freezing samples, culling animal colonies, and moving conferences online [6-8]. Universities rapidly shifted course content online $[9,10]$. However, the initial focus of supporting principal investigators to close their laboratories and helping undergraduates transition to online learning left many graduate students and postdoctoral fellows feeling like afterthoughts in the early days of COVID-19.

More recently, there has been increased awareness of how the COVID-19 research shutdown will impact graduate students and postdoctoral fellows [11-13]. Most of this work has focused on using past data about this population to predict what difficulties and challenges they will experience due to laboratory closures. Some groups have asked graduate students directly what their concerns are and what supports they require, including work from Australia showing an increase in financial hardship amongst $\mathrm{PhD}$ students at the University of Sydney [14].

The purpose of this study was to examine the impact of research disruption during the COVID-19 pandemic on graduate students and postdoctoral fellows completing laboratory-based research in Canada. We did this through a national online survey asking respondents about events surrounding laboratory closures, activities completed while working from home, and their concerns about how COVID19 will impact them going forward. Our aim was to identify areas of support needed by graduate students and postdoctoral fellows during the COVID-19 pandemic, as well as support needed once the pandemic dissipates. We also wanted to identify best practices for widespread laboratory shutdowns that could be used if similar situations arise in the future. In addition to identifying these supports and best practices, we have documented the stages of grief experienced by graduate students and postdoctoral fellows in response to the COVID-19 laboratory closures.

\section{Methods}

\section{Ethics Approval}

The study protocol was approved by the Hamilton Integrated Research Ethics Board (HiREB) under project number 10832. Following the recruitment of potential interview participants, a protocol amendment was made to have an additional interviewer, as the primary interviewer had a conflict of interest with two participants. Correspondence with HiREB indicated a formal addendum was not required. Instead, a note to file was added to the study file to document this protocol modification.

\section{Study Design, Participants and Recruitment}


Two parallel methods were used to collect data: an anonymized online survey and an optional follow-up one-on-one virtual interview. The target population for this study was all persons who:

(i) are a graduate student or postdoctoral fellow in Canada, (ii) engage in laboratory-based research, (iii) had their research stopped or paused by the COVID-19 pandemic. There were no inclusion or exclusion criteria based on age, ethnicity, disability, gender, or race.

The online survey was promoted via Twitter and email. For email advertisements, the investigators contacted coordinators of email subscription lists including departmental administrative staff, graduate student associations, postdoctoral fellow association, and professional academic organizations. These individuals or groups were selected due to their ability to share study information widely with graduate students and postdoctoral fellows.

The online survey was administered through LimeSurvey, taking approximately 30-45 minutes to complete. Participants contacted by email received a letter of information about the study in PDF format, and a link to access the survey. Participants contacted via Twitter were provided a link to access the survey, where an electronic version of the letter of information was provided prior to obtaining consent to participate. All survey questions were optional, with respondents permitted to skip any questions they preferred not to answer. The online survey was open from April 27, 2020 to June 8, 2020, with 315 respondents completing at least one section of the survey.

At the end of the survey, participants could opt-in to participate in one-on-one virtual interviews and/or in follow-up surveys following dissipation of the pandemic. Those interested in participating in virtual interviews were sent a separate letter of information outlining the survey protocols.

Semi-structured interviews were conducted from May, 112020 to June 8, 2020 with 18 participants via Zoom, a video conference software that allows for the recording of meetings. Interviews lasted between 30 minutes to 1 hour. Participants' cameras were turned off during the interview process. Following the transcription of interviews, video recordings were deleted.

\section{Data Analysis}

Survey and interview data were formatted and transferred to the qualitative data analysis software MAXQDA (VERBI GmbH, Berlin, Germany). Descriptive statistics were generated for both data sets. Quantitative data was entered into GraphPad Prism 8 (GraphPad Software, San Diego, USA) for analysis and formatting.

A thematic analysis was conducted of the narrative feedback from the surveys. Themes were discussed and refined by two researchers. The interview transcripts were then analyzed using these same themes, as the interviews were designed to drill down into information provided on the surveys. Data from interviews was used to (i) confirm themes from the survey and (ii) to provide more specific details on individuals' experiences. Beyond information on specific lab shut down requirements (for example, 
differences between animal labs and frozen sample labs), no new themes emerged. Data from individual interviews did not encompass all themes, but collectively, all themes were addressed. The researchers analyzing the data debated the granularity of themes. More granular themes were more useful and provided a clearer picture of the survey data, while more general categories were more useful when considering the interview data collectively. With these caveats, there were no noteworthy discrepancies between the two data sets. These themes were given to an independent third investigator to ensure identified themes could be subsequently found by someone who had not previously seen the data. A full list of themes can be found in Supporting Table 1.

As was expected, some of the data collected in the interviews was highly specific to individual circumstances. While this did not result in additional themes, this information is important to consider. It draws attention to the fact that laboratory shutdown and reopen protocols must account for local, specific circumstances. While generalizations can be made, there is no one-size-fits-all laboratory shutdown (or reopen) template. All plans must account for laboratory-specific scenarios.

\section{Results}

\section{Respondent Characteristics}

The online survey was completed by 315 respondents (Fig 1). Biology (30.2\%) and Health Medical Sciences (19.4\%) were the most commonly reported respondent area of research (Fig 1a). Eight provinces were represented, the majority from Ontario (34.9\%) and Quebec (17.1\%) (Fig 1b). Most respondents lived with at least one other person while working from home, with only $17.6 \%$ of respondents living alone (Fig 1C). Slightly less than half of respondents lived with a partner or spouse $(44.1 \%)$ and $23.9 \%$ reported living with parents (Fig 1C). Half of the respondents were female, $18.4 \%$ male, and $1.2 \%$ were non-binary or genderfluid (Fig 1d), with just over a quarter of respondents (27.9\%) not disclosing their gender. PhD students made up $37.5 \%$ of respondents, with Master's students representing $21.6 \%$, postdoctoral fellows representing $14.9 \%$, and $26 \%$ not disclosing their position (Fig $1 \mathrm{e}$ ). There was a fairly even distribution of graduate student respondents with regards to degree progress, with fewer students in the process of defending their thesis (Fig 1f). Postdoctoral fellow respondents ranged in experience from less than 12 months to greater than 48 months in their current position (Fig 1g).

A slight majority of respondents (59.6\%) had previously published research articles (S2 Table). Postdoctoral fellows were more likely than graduate students to be looking for their next academic position, with $57 \%$ of postdoctoral fellows seeking a new position compared to $28 \%$ of Master's and $23 \%$ of PhD students (S2 Table).

The 18 interview participants reflected general overall demographic characteristics to that of survey respondents (S3 Table). Most interviewees were female (66.6\%), PhD students (72.2\%), conducting biology (38.9), or health and medical science research (33.3\%) (S3 Table). Four Canadian provinces were represented, with the majority of interviewees being from Ontario- $(50.0 \%)$ or Quebec (38.9)-based 
research institutions (S3 Table). Similar trends were seen in degree progress, the number of months in a postdoctoral position, and those seeking new academic positions (S3 Table).

\section{The Transition from Research to Social Distancing}

We asked survey respondents who made the decision to pause experiments or close down laboratories. Seventeen percent reported they personally made the decision to stop experiments before their laboratory was closed (Fig 2a). Twenty percent had supervisors who closed their laboratories prior to an official shutdown (Fig 2a). Of the remaining respondents, $45.6 \%$ experienced closures decided by their department, research institute, faculty, or university (Fig 2a). The majority of respondents last completed experiments in March 2020, with 68.4\% ending in between March 8, 2020 and March 21, 2020 (Fig 2b). Only $13.4 \%$ came from laboratories with standard operating procedures (SOPs) for quickly shutting down the laboratory created prior to the COVID-19 pandemic, with an additional $18.1 \%$ laboratories creating a shutdown SOP in response to the pandemic (Fig 2c).

Respondents referenced a lack of clear and consistent communication between supervisors, administration, and trainees leading to a chaotic and confusing shutdown experience. Changes in protocol happened quite suddenly, as two respondents explained:

"One day, we were told that we could continue recruiting human subjects; the next, everything was to be shut down" (Survey ID 770)

"After a week of emails saying like, 'We're open. We're open. We're open.' [...] And then that night around 6:30 PM, after getting an email at 3 p.m. saying 'We're open', we got an email saying 'Gotcha. Campus is closed'." (Interviewee 6)

There were also discrepancies among people in positions of power in how they reacted to the impending shutdown. This led to graduate students and postdoctoral fellows discussing the situation amongst themselves, increasing their anxiety as many did not have the positional power to enact change at the laboratory or institutional level:

"Some people in charge were behaving like this was a minor inconvenience and others were behaving like this was an apocalypse, you know? So I think having those kinds of contradictory messages from people in charge was difficult." (Interviewee 4)

"There was discussion and there was lots and lots of uncertainty and anxiety, I would say, from myself and my peers. Looking towards our supervisor was not very helpful, as I felt that we were more informed about what other institutions were doing than they were." (Interviewee 11)

Concerning the logistics of laboratory and project shutdown, respondents reported multiple steps to pause projects including: backing up data, setting up remote servers to access data, discontinuing ongoing experiments, freezing samples for indefinite storage, culling animal colonies to reduce numbers 
or sacrificing animals early to obtain data, discarding samples that couldn't be stored long-term, securing equipment and biohazards safely, rescheduling or cancelling in person meetings with human participants, cleaning laboratory space, testing that back-up power supplies to key equipment (such as freezers) were functional, turning off non-essential equipment, and the physically locking up the laboratory. Twenty-nine percent of respondents helped shut down others' projects in addition to their own (Fig 2d). A respondent's academic position did not significantly impact the likelihood of assisting in other shutdown activities outside of their project ( $\mathrm{S} 4 \mathrm{Table}, \mathrm{P}=0.2896$ ).

When asked if, in hindsight, they would change anything about the shutdown procedure, respondents discussed three main things they would do differently. First, they would stop experiments sooner out of their own volition rather than being abruptly cut off by the university. Second, they would want "more clear communications from everyone involved" (Survey ID 704). Third, they would have brought more things with them from the lab, including personal belongings, data, technology, and print resources. Many did not initially take these items as they "did not think [they] would be locked out so long" (Survey ID 156). This also included setting up remote access to data and laboratory information.

A slight majority (63.7\%) of laboratories had a uniform closure, meaning all students and fellows stopped experiments at approximately the same time (Fig 2e). A similar trend was also reported at the departmental level, with most (70.1\%) laboratories closing in unison (Fig 2e). A frequent reason cited for an staggered closure of laboratory experiments was differences in ease of pausing experiments. For example, many respondents were understanding that animal model research might take longer to pause:

"Those who had to finish animal data collection went into lab while others started working at home. It made a lot of sense." (Survey ID 158)

"My colleague was in the middle of an animal experiment and had she terminated the experiment early, she would have had to sacrifice animals without collecting any data, rendering those animals a waste. I am glad she continued the experiment until the planned end-point, thereby not having to use any additional animals." (Survey ID 188)

On the whole though, respondents who started to work from home while their colleagues continued working in the lab had a mix of responses to the situation. Many wished they could have continued working for longer, feeling "Frustrated and angry that [colleagues] would complete their research while I had to start over" (Survey ID 183) or that "It felt unfair and I was a little jealous" (Survey ID 253). This mainly stemmed from the thought that they were falling behind compared to their peers who had stayed behind. Others were upset specifically that they had been the ones told to go home first, as this was interpreted to mean their project had "lower apparent priority (Survey ID 117) compared to others in the laboratory. There was also feeling of concern for colleagues who continued working, as one respondent explained:

"It was also worrying to see others still complete work and putting themselves in harm's way during a pandemic to ensure they could actively finish their experiment." (Survey ID 18) 
On the other side, respondents who continued working longer than their colleagues reported feelings of anxiety and guilt. Some felt "guilty that I was putting others at risk" (Survey ID 22) by continuing to work in the lab and that the concern of their colleagues made them feel more anxious. As summarized by one respondent, "I was the one working. And felt like I was doing something wrong" (Survey ID 325).

Respondents who experienced more uniform laboratory closures had different sets of reactions to the research shutdown. Similar to those who had uneven closure, many felt "anxious because it was really starting to show the reality and seriousness of the situation" (Survey ID 284). However, this anxiety stemmed from realizations that COVID-19 was significant enough to warrant large scale closures, rather than concern about their safety or the safety of colleagues. There were also conflicting feelings of isolation and unity. On one hand, respondents reported feeling "Lonely and sad. I really miss being around my peers" (Survey ID 425). This stemmed largely from losing in- person social interactions. On the other hand, respondents said "it created a sense of solidarity for me. Everyone was working together to try and keep people safe" (Survey ID 533). Others cited a uniform closure as bringing an end to the confusion and mixed messages they experienced prior to the shutdown, "I think I felt a bit relieved when all the labs finally shut and I knew that everyone was at home. It just meant that all the uncertainty of when the labs were going to close was over and everyone was now on the same page" (Survey ID 767)

We asked respondents to identify if they received any pressure to continue experiments prior to receiving an official shutdown directive from their research institution, and if yes, the source of this pressure. The majority of respondents indicated that they received no pressure to continue from their supervisor (65.6\%), peers within the laboratory (74.4\%), and peers external to the laboratory (84.0\%) (Fig 2f). Those who were pressured to continue experiments were more likely to be pressured by persons in their laboratory group, with one third being pressured by supervisors and one quarter by peers within the laboratory (Fig 2f).

Some respondents who experienced external pressure said their supervisor "does not believe COVID is a very serious issue and that responses have been an over-reaction" (Survey ID 265) or that they "kept saying things like 'you can't just STOP research"' (Survey ID 364). For others who had peers who "suggested that we were overreacting " (Survey ID 255) or who "felt like the pandemic wasn't super serious" (Survey ID 515), these respondents would question whether shutting down laboratory experiments was the best decision.

A much different trend emerged when respondents were asked about pressure they put on themselves to continue research: $70.7 \%$ of respondents reported some level of internal pressure to continue working (Fig. 2F). This is consistent with well known levels of stress caused by the publish-or-perish research environment and the activities students and fellows undertake to do well in such environments [15-18]. Respondents described fear of losing research time and data, often tied to a fear of not being able to graduate or submit a manuscript:

"My thesis is due at the end of the summer. I was scared because even if research were to have started up again, I lost my window of time where I could have continued research. At this point, I just have to write a 
thesis with the data I have. " (Survey ID 73)

"I am losing a lot of valuable data collection time and am concerned I won't finish on time to graduate." (Survey ID 417)

"The days prior to the shutdown were filled with some pressure because I was trying to finish experiments that would provide data for an upcoming manuscript. My hope was that I'd get the data I needed and then go home and start writing the paper. " (Survey ID 71)

Others explained they felt self-conscious stopping work when peers were working, worried that they would be perceived as less hard working or lazy.

Following laboratory closures, a majority of respondents $(79.2 \%)$ remained in the same city in which their laboratory is located (Table 1), with the most common explanation that it is the location of their full time residence. Other reasons to remain included "not wanting to do unnecessary flying" (Survey ID 99), international flights being cancelled, and not wanting to put family or friends at risk by traveling from an area with a high incidence of COVID-19. For the fifth of respondents who relocated to another city, most cited moving to be with family as a primary factor, many because they lived by themselves and did not want to be alone or because they wanted to move to "an area with fewer cases and less dense population" (Survey ID 739). A minority of respondents did not initially plan on moving, but due to how quickly shutdowns occurred, they chose to stay where they were when they were informed of laboratory closures:

"I had gone home to visit my parents for a weekend, and they live in another city, and I thought it was just going to be for a weekend, but then the conversation [concerning laboratory closures] shifted over the course of the weekend. So instead of coming back to the big city and the densely populated area, I stayed behind." (Interviewee 2)

\section{Working from Home during Social Distancing}

Following the closure of the laboratory work space, $48.6 \%$ of respondents had individual workspaces in their homes, while $31.0 \%$ shared workspace with others in their household (Table 2). $20.4 \%$ of respondents did not have a dedicated space in their home to do work (Table 2). $21.9 \%$ of respondents were taking one or more courses as part planned studies which were transitioned online (Table 2). Approximately a third of respondents had one or more projects in the process of peer review (Table 2).

Respondents completed a number of activities while working from home, which we classified as being directly related to COVID-19, self-care, research, or household maintenance (Fig 3). More respondents reported participating in COVID-19-related activities that directly impacted their family or friends as opposed to the public at large. For example, $76 \%$ of respondents indicated sharing accurate information about COVID-19 with family and friends, compared to $19 \%$ sharing the same information with the general public (Fig 3). Other COVID-19-related activities included donation of personal protective equipment 
(10\%), volunteering to support COVID-19 research (11\%), and supporting others in quarantine (45\%) (Fig $3)$.

Respondents reported high levels of self-care activities while working from home (Fig 3). Many prioritized connecting with family and friends virtually, with $84 \%$ connecting by video chat, $86 \%$ using a text-based medium such as email or texting, and $80 \%$ using phone calls (Fig 3). Exercising either indoors or outdoors was also frequently reported (85\%) (Fig 3). While $74 \%$ respondents returned to previous hobbies or skills, $43 \%$ of respondents said they began developing a new hobby or skill while working from home (Fig 3 ). Other self-care activities with lower levels of reporting included establishing a new routine (48\%), meditation or mindfulness (30\%), and attending virtual religious or spiritual services (9\%) (Fig 3).

Despite being away from the laboratory environment, respondents continued to participate in a number of research-related activities, the most common being reading the literature (85\%), virtual lab meetings (82\%), and analyzing data gathered prior to shutdown (61\%) (Fig 3). Writing-related activities such as drafting manuscripts (53\%), designing scientific figures (42\%), and writing thesis chapters (33\%) were reported (Fig 3 ). Slightly less than half (45\%) of respondents attended virtual scientific meetings, conferences, or seminars from home (Fig 3). Other respondents did activities to help prepare for after the pandemic, such as creating strategic plans for future experiments (44\%) or updating their curriculum vitae and/or Linkedln profile (43\%) (Fig 3).

There were high levels of reported household maintenance activities, including cleaning tasks (94\%), cooking meals for self (89\%), cooking for others (70\%), and completing necessary excursions such as grocery shopping (80\%) (Fig 3). In terms of caregiving, $10 \%$ of respondents reported caring for children, while $3 \%$ reported caring for dependent adults (Fig 3). $39 \%$ of respondents reported caring for pets (Fig 3 ). Overall, more than half of respondents thought there was an even distribution of labour in their household (66.1\%) (Table 3). Slightly more men than women reported that household labour was evenly distributed (76.6\% vs 63.2\%), however this trend was non-significant (Table 3, $\mathrm{P}=0.11$ ). Many respondents described "dividing tasks so that everyone plays a role and contributes to household chores" (Survey ID 87).

Others reported one or more members of the household intentionally took on more household responsibilities from others:

"My partner isn't employed right now so he does more than half the chores because he has more time than me." (Survey ID 29)

"Some household members have chronic health issues and aren't able to do as much." (Survey ID 462)

However, some respondents had an uneven distribution of household labour that they were dissatisfied with, describing a continuation or amplification of an imbalance which existed prior to the COVID-19 pandemic:

"Some gender roles are hard to escape and I find myself, now that I am always home, picking up even more of the slack than before." (Survey ID 248) 
"Though my partner and I both work from home, I still feel like a lot of home duties fall on me. I do $80 \%$ of the cleaning and cooking-though this is not much different prior to COVID." (Survey ID 741)

Respondents were asked to rank how frequently they experience, if at all, a variety of symptoms of distress while working from home (Fig 4). We used a modified list of distress symptoms (Items 1-8, S4 Tale) previously developed by the National College Health Assessment Survey II (NCHA II) [19]. Additional items (Items 9-16, S5 Table) were included as there was frequent anecdotal reporting of these symptoms by graduate students through social media. The most commonly reported symptoms related to attention, including lowered productivity and difficulty focusing on tasks (Fig 4). Other symptoms with high incidence included difficulty getting out of bed, exhaustion not from physical activity, and feeling overwhelmed (Fig 4).

A greater proportion of female respondents reported symptoms of distress than male respondents (S5 Table). However, only two distress items had significant differences between men and women; feeling overwhelming anxiety $(P=0.0067)$, and being easily annoyed or irritable $(P=0.0029)$ ( $S 5$ Table). This trend is consistent with past findings and literature showing women are more likely to report symptoms of distress [20-22].

Respondents were asked to identify their three biggest barriers to getting work done at home, to which four main barriers were identified. The first barrier was technical issues, which primarily stemmed from lack of access to stable, high speed internet. Other issues in this category included not having enough computer processing power to run analyses and access to software. The second barrier was distractions in the home, such as noise, other household members, and household chores that are not present in the laboratory:

"So if I just get up to go grab a glass of water, then I'll see something and I'll fix it. Or oh, there's a little bit of dishes. I'll start doing them and I'll start doing other things, and I'll never go back to work. So when I work in the lab and in my office at the desk, there's nothing else." (Interviewee 14)

Respondents with children also cited child care as another distracting factor, as one respondent put it "[My daughter] is only 15 months so she requires a lot of hands on attention, so my husband and I take turns watching her so we only work for 2-2.5 hours/day" (Survey ID 137).

The third barrier identified was motivation, or lack thereof. Respondents had difficulty starting tasks, focusing on tasks, and finished activities. Some attributed this to lack of impending deadline, as "nothing seems pressing and I sometimes genuinely do not feel like working" (Survey ID 129). Others felt that their "research is less relevant now" (Survey ID 52), and since they were unable to do experiments they lacked motivation to do other activities.

The fourth barrier was mental health, with either new anxiety or depression developing or previous conditions worsening with the pandemic shutdown. As one respondent explained "managing my anxiety and depression during this uncertainty has left very little energy to focus on work " (Survey ID 311), thus 
their main activity during this period was taking care of themselves. Oftentimes, lack of motivation and mental health barriers compounded with one another, as described by one respondent:

"My brain seems to just shut down all the time, and I have a hard time controlling and coming out of it. I just feel so overwhelmed thinking I have so much to do that I don't do anything and then I get anxious because I'm afraid that I didn't do enough work." (Survey ID 562)

Next we asked respondents to list three supports they received while working at home, to which five main categories of support were identified. The first support was financial, with respondents citing the "financial security of my stipend" (Survey ID 311), the Canada Emergency Student Benefit, the Canada Emergency Response Benefit, and other sources of monetary support in order to cover costs and "prevent financial anxieties" (Survey ID 48). The second support was social connections, which was described as coming from a number of sources, including family, friends, a partner or spouse, children, laboratory peers, and pets. As summarized by one respondent, these social connections made them "feel supported and validated in my feelings, and knowing that we're all struggling. Support from friends and peers makes me feel less alone and I know I'm not the only one having trouble with this" (Survey ID 493).

The third support was establishing and maintaining routines during social distancing. Respondents said having routines, either self-imposed or from external sources such as a weekly laboratory meeting, helped with finding motivation, as well as "feeling that there is a rhythm and purpose to my daily life again" (Survey ID 133). Dog-owning respondents cited their pet, in addition to emotional support, also served as an external source to gently force them to maintain healthy routines:

"Having a dog has helped me stay in a normal routine of getting up at a decent time to take her outside and getting out for a walk every day." (Survey ID 515) "My dog insists on thrice daily walks, which gets me up and moving. And I find that I get back from walks and I can focus a lot better." (Interviewee 6)

The fourth support respondents identified was relating to mental health, which mirrors the previously identified barrier. This included professional support, including counselling, therapy, and remote telemedicine services. Other supports included meditation and exercise as means to maintain mental health. As explained by one respondent, "running removes all the excess anxiety and improves my mood" (Survey ID 116).

The fifth type of support came from the respondent's supervisor or other administrative figure. This included virtual laboratory meetings to implement routine as well as keeping respondents socially "connected to my work and my co-workers" (Survey ID 255). However, most respondents identified supervisors' and administrators' understanding, transparency emotional support as what they valued the most:

"My supervisor is very supportive not only of my research but also my well-being. He listens and encourages me to take care of my mental health and understands that this social distancing thing is 
having a pretty big impact on me." (Survey ID 562)

"[My] supervisor telling the group during lab meeting to focus on health and family and not expecting any productivity at this time." (Survey ID 87)

"My department chair has been very transparent which has provided clarity and some ease of anxiety." (Survey ID 195)

"I'd say my supervisor genuinely, she has been incredibly supportive during this time of understanding what it's like to go through this, but also keeping us very aware of decisions that are being made and very realistic." (Interviewee 3)

\section{Concerns and Supports for Returning to the Laboratory}

Respondents had a number of concerns that the COVID-19 pandemic could impact them going forward (Fig 5). These worries tended to fall into three categories: personal, impacting research activities, and impacting career. A minority of respondents also said they were not very worried that they would be impacted by the ramifications COVID- 19 .

As personal concerns, respondents were worried friends or family members would get sick or die from COVID-19, especially persons who had underlying health conditions or were essential workers. Interestingly, respondents were more worried about others contracting COVID-19 than themselves (Fig 5). Respondents were more concerned about "the impact COVID-19 will have on my long term physical and mental health" (Survey ID 516). Thus, the concern came from the impact of social distancing and working from home in response to COVID-19, rather than concern of contracting the virus.

Related to the concern of mental health were concerns surrounding decreased social interaction while working from home, which left them "feeling lonely and isolated" (Survey ID 394). Some did not know when they would be able to see their family face-to-face again. Others were concerned that this would negatively impact their ability to make connections with people going forward as "spending so much time in social isolation will just exacerbate" (Survey ID 513) their natural awkwardness or introversion. Maintaining current social connections was also a challenge, as one respondent put it:

“Not being able to physically see people makes it harder to maintain connections. I had a 'COVID' birthday and it felt like I was forgotten by most people, even though a birthday is not a priority in times like these." (Survey ID 37)

A section of respondents were concerned that they would need to delay key life milestones such as getting married, beginning a new job, or starting a family. Often these events were pushed back due to a respondents' graduation date or end of postdoctoral fellowship getting extended due to laboratory closures. One respondent summarized the impact of COVID-19 as follows: 
"This situation has screwed up my whole life plan, I just want to scream, I feel like I have no control." (Survey ID 377)

Despite financial help being identified previously as a support by some respondents, $56.6 \%$ worried that COVID-19 would impact their personal finances. Some were struggling even with governmental support, others were worried about the security of their research funding. Increased financial uncertainty was cited as a reason some respondents were choosing to delay life milestones. Others were frustrated that they needed to continue to pay tuition fees, despite not being able to conduct research:

"I am still paying full tuition to sit on my couch and not be allowed into my lab, when that tuition I'm paying is to be a part of a research program, a research program I currently cannot take part in." (Interviewee 17)

Respondents listed a number of concerns related to their current research activities. Many were worried "that funding for basic research will become scarce"(Survey ID 80) due to the focus on COVID-19 research and the state of the economy. Others were concerned about going overtime on their degree or fellowship caused by COVID-19 would impact their eligibility for funding in the future. Traditionally, this would result in reduction or stoppage of stipend money. Although many institutions and funding agencies have announced accommodation for students and fellows for this fall, respondents were more concerned about the "availability of funds next year and the coming years, [as] that may actually be an issue" (Interviewee 8). This worry of going overtime or delaying graduation due to decreased research productivity was intertwined in a number of responses throughout the survey. Some respondents had "cancelled [their] current research topic and forced [them] to have to change it" (Survey ID 664) in response to the laboratory shutdown, modifying their thesis in a way that would forever be impacted by COVID-19. Others lamented canceled travel opportunities for networking and "missed opportunity for international collaboration" (Survey ID 742). As one respondent explained:

"I'm in that crucial new investigator period of my career. COVID represents a year of no conferences, no networking, no data collection... it's setting everything back." (Survey ID 203)

Many concerns regarding current research activities connected with concerns about how COVID- 19 might impact their careers: $86.4 \%$ of respondents were worried about their competitiveness as a researcher, with $36.6 \%$ describing themselves as 'extremely worried' (Fig 5). Many respondents were concerned that their lack of productivity during the laboratory closure period and decreased publications would make them less desirable candidates for future positions. As summarized by one respondent,

"My goal is to become a tenure-track faculty member at a research-intensive university, which is a difficult enough task as is. But I worry that because of this situation, I will either not be productive enough to secure a position, or I will have to prolong my postdoc experience in order to gain enough productivity to be a viable candidate. I also worry that universities will have decreased hiring in the next few years, which coincides with my entry into the academic job market." (Survey ID 255) 
Many respondents were concerned about the availability of employment both within and outside academia in the post-COVID-19 era . Some hope "there are still jobs in academia (or any kind of research) after all the economic fallout" (Survey ID 99). Postdoctoral fellows, in particular, were concerned about universities freezing hiring for faculty positions. Some respondents had already made the decision to alter their career goals in response to COVID-19:

"I am convinced about leaving Academia." (Survey ID 113)

"Before COVID, I was gaining momentum in my project. I was deciding between transferring from MSc to PhD, but after COVID I lost this motivation entirely. I now have decided to finish with an MSc and hopefully find a job afterwards." (Survey ID 763)

"I feel like the time away from [the lab], whether this is good or bad has maybe made me cooled off a little bit and like feeling more, I don't know, further from science and thinking that maybe that's not bad." (Interviewee 4)

Respondents had a number of ideas when asked about what supports would help them transition back to laboratory work post COVID-19 which we've categorized into five groups: personal protective equipment and protocols, understanding and empathy, guidance and direction, timeline support, and financial support.

The minimum support respondents asked from both supervisors and administration was adequate personal protective equipment and protocols on how to reopen and work in laboratory space. The emphasis was placed on clearly communicating safety procedures and protocols that are put in place, as respondents did not want a similar confusion and anxiety that took place during laboratory shutdowns to repeat themselves.

What respondents wanted most from their supervisors was the first two groupings of support: (i) understanding and empathy and (ii) guidance and direction. First, respondents wanted "understanding I won't be as productive as before COVID for a long time" (Survey ID 458), "reassurance that delays are acceptable" (Survey ID 739), and "emotional support and encouragement" (Survey ID 769). Respondents described wanting their supervisors to be explicit that they do not expect pre-COVID-19 levels of productivity and that they will not be made to feel guilty over lost time. They also wanted "moral support getting back on track with experiments" (Survey ID 123), as many respondents are still reeling from the emotional and stressful toll of the shutdown experience. Respondents described hesitancy and fear at returning to laboratory situation where this flexibility was unlikely to be found:

"My supervisor has zero empathy or tolerance and I'm extremely worried about going back to work with him" (Survey ID 69)

Second, respondents were hoping to receive explicit guidance and direction from supervisors as to where to go with projects once they returned to work. There were frequent descriptions of wanting "clear goals for me to finish my program" (Survey ID 93), "a detailed plan of how to be most efficient with research 
efforts" (Survey ID 347), and "clear communication" (survey ID 248). Respondents encouraged the development of a research plan that would limit the potential negative impacts of the laboratory shutdown, get experiments back up and running efficiently, and keep respondents on time in their degree progression. Respondents also wanted supervisors to "acknowledge that the $\mathrm{PhD}$ or the master's degree that students had originally set out to accomplish may not be feasible within our current time frames" (Interviewee 11) and that adjustments would be required.

The supports requested from administration, including departments, faculties, and the university itself, focused more on structural supports including degree timeline and financial supports. Timeline support focused mainly on flexibility. Some students explicitly asked for "flexibility for certain milestones (comprehensive exams, graduation timeline, funding timeline) that allows us to get back to our research without feeling like we need to compress everything into the time that we lost" (Survey ID 92). Others wanted expectations for key requirements to change as extending time in their degree "is something I really don't want to do" (Survey ID 651) and wanted "assurance that I will graduate on time" (Survey ID 583). Respondents had a variety of suggestions for financial support, including increasing fundable periods for all students and fellows, ensuring students who go overtime due to COVID-19 are not penalized financially, lowering the cost of tuition, and increasing financial support for students to purchase software and technology to work partially from home. Respondents also asked that specific guidelines for timeline and financial support be developed for international students and fellows. This was due to international trainees having a unique set of concerns regarding these two items related to Canada's immigration and visa policies, which could potentially change in response to COVID-19.

\section{Grief Response to COVID-19 Laboratory Closure}

There was an underlying feeling of grief that permeated throughout survey and interview responses. Respondents lamented the data that was lost, the conferences that might have been, and how this new reality of social distancing and working from home was not something they ever expected. We used the Kübler-Ross model of grief as a lens to view respondents' reactions to the laboratory closures.

The Kübler-Ross grief construct is one of the most well known models to describe the process of grief [23]. Although initially developed to describe how people process the grief that results from terminal illness and death, it has also been used to describe grief from a variety of sources including job loss, educational reforms, and organizational change [24-27]. The Kübler-Ross model outlines five stages of grief: denial, anger, bargaining, depression, and acceptance. The stages described by Kübler-Ross are not a linear process, but can occur simultaneously and in any order [23].

Denial is the disbelief following unexpected and shocking news, which Kübler-Ross and others posit gives individuals time to process $[23,28]$. Anger is when frustration boils over, often due to perceived unfairness of the situation [23]. Bargaining focuses on what could have been done differently to change what is 
happening, even if nothing could have been done to prevent the situation [23]. Kübler-Ross described bargaining as the temporary belief that "there is a slim chance that [the person experiencing the change] may be rewarded for good behavior and be granted a wish for special services", that they will be 'rewarded' for this behaviour and the situation will be better than anticipated [23]. Depression is the sense of loss that occurs when the person acknowledges the situation can not be 'fixed' or glossed over, replaced by a sadness that is often very private [23]. Acceptance is learning how to live with the new reality the person finds themselves with, finding a new equilibrium that does not have the same emotional highs and lows of the previous four stages [23]. Some scholars suggest 'hope' could be a sixth stage to the Kübler- Ross model, however, Kübler-Ross suggests hope is present throughout all stages of grief and simply reasserts itself during acceptance [23].

Respondents were provided with a list of 37 randomized feeling words, each of which has been previously attributed to a stage of the Kübler-Ross model [29]. They were asked if they had experienced any of these feelings during the laboratory shutdown period, while working from home, and when they thought about the future (Fig 6). Respondents could also include other emotions they felt during these periods, which were then classified into Kübler-Ross stages based on categorization by Kearney and Clapper [29,30].

Anxiousness was highly reported across all three timepoints, mirroring the symptoms of distress reported in Figure 4 (Fig 6a). The trend of anxiousness reflects most feeling words in the bargaining stage, showing a decrease in reporting at the second time point and increasing in the third (Fig 6a). Conversely, depression stage feelings like "lonely", "discouraged", "depressed", or "apathetic" had higher levels of reporting while respondents were working at home compared to other time points (Fig 6a). A similar trend was found with "calm", "contented", and "easy-going", three acceptance stage feelings (Fig 6a). More subtle trends included the reporting levels of "disbelieving" decreasing at each subsequent time point (Fig 6a). Two feeling words with the opposite trend were anger, bitterness, and "afraid" both of which saw the highest levels of reporting when respondents were asked to think of the future (Fig 6a).

When observing Kübler-Ross stages as a whole, rather than individual feeling words, more respondents report bargaining-associated feelings across all three timepoints (Fig 6b). The second most-reported feeling category was depression, which saw an increase in self-reported feelings during the work from home period (Fig $6 \mathrm{~b}$ ). The three remaining Kübler-Ross stages have more subtle variations, with overall denial decreasing, anger increasing, and acceptance having a similar trend to depression (Fig 6b).

Although the majority of respondent feedback focused on negative feelings deriving from being in a pandemic, there were small undercurrents of optimism for the future. With time and distance from the laboratory shutdown, these may grow further. As succinctly pointed out by one respondent: "I really hope it's all going to be okay" (Survey ID 265).

\section{Discussion}


In this study, we assessed the self-reported impact of the COVID-19 laboratory closures on graduate students and postdoctoral fellows. This was done through a mixed-methods analysis of online survey data from 315 unique respondents and 18 semi-structured interviews. Some data collected was highly specific to individual respondents and laboratories, emphasizing there is no one-size-fits-all shutdown or reopening protocol. The distinctive characteristics inherent to each laboratory across Canada will require any plans made to account for their unique context. However, some key generalizations can be made, rooted in experiences, feelings, and concerns shared by respondents across the country. These findings also align with those found in a larger nationwide survey of graduate students, which included respondents of non-research based and professional programs [31]. A summary of fundamental recommendations derived from our findings are summarized in Table 4.

Respondents' experiences of initial laboratory shutdowns were, for the most part, chaotic, confusing, and abrupt. Institutional and laboratory policies changed quite rapidly, including the decision whether to close research laboratories at all. This uncertainty mainly stemmed from a lack of clear, consistent communication from administrators. The lack of consistency in policy within research institutions, as well as between institutions, left many graduate students and postdoctoral fellows feeling anxious and adrift. Much of this is understandable, as administrators themselves were reacting to an unanticipated and emerging situation for which they did not have prior experience to inform them how to proceed. However, this uncertainty was compounded as information and decisions were released, from administrators to principal investigators to graduate students and postdoctoral fellows. If similar situations arise in the future, minimizing discrepancies in messaging, as well as abrupt changes in policy, will minimize potential harms and stresses on graduate students and postdoctoral fellows.

In terms of the physical closure of the laboratory space, respondents had a clear understanding of what was required to pause their research projects in terms of preservation of data and safety procedures. When asked what they would do differently if they had to repeat the closure experience, most focused one beginning the shutdown earlier and bringing more materials home with them. Both of these, as well as the desire for better communication from administrators, stem from an underestimation of the gravity of the COVID-19 pandemic and how long the laboratory closure would last. Many lamented limited access to data and laboratory notebooks. One solution that some laboratories implemented was setting up remote access to laboratories resources, software and data. Although there was a technological requirement needed to support this remote access, respondents who identified that they were able to access data remotely found it valuable. This strategy could be used by others to preemptively prepare for future laboratory closures.

Another factor to consider for future laboratory shutdowns is the decision to end all work at approximately the same time, or to allow some laboratory members continue working during a partial shutdown. Graduate students and postdoctoral fellows who experienced uniform and staggered laboratory closures had distinct emotional responses to the shutdown. Although both groups experience anxiety, the root of the anxiety came from different sources. For those from staggered closures, this unease came from fear of falling behind in data collection for those who were at home, and fear of 
putting themselves or others at risk from those who were in the lab. By contrast, respondents from uniform closures cited a realization of the seriousness of the situation as the source of their anxiety. Another key contrast is that respondents from uniform closures reported a sense of safety coming from the solidarity and unity of everyone stopping work at once, which did not occur for those with staggered closures. This suggests that a uniform approach to laboratory closures should be used whenever possible, with exceptions for certain types of experiments which take longer to pause such as animal model research. Not only will this minimize negative outcomes for graduate students and postdoctoral fellows at the time of the closure, but prevent a hierarchy between those with 'high priority' and 'low priority' projects from emerging in the long term.

Overall, research institutions should proactively plan for their future pandemic response, rather than have another reactive situation like that which was experienced during the COVID-19 laboratory closures. Developing official guidelines on pandemic responses, similar to other emergency situation response guidelines which are already in place will help promote consistency in implementation and minimize potential chaos or confusion. It will also reduce the need for abrupt changes in policy and sudden closures.

Graduate students and postdoctoral fellows reported completing a variety of activities while working from home. This included research-related activities, despite not having access to the laboratory space. Unlike previous research, $66.1 \%$ of respondents believed that there was an even distribution of household labour. For some this distribution was intentional, in order to assign tasks based on free time availability. For many, however, it was not, citing gender roles regarding domestic tasks and caregiving as one contributing factor. This in part could explain some of the trends we see, as the majority respondents did not report child care responsibilities. The challenges of parenting while conducting research, especially for female researchers, has been previously documented in the literature and has been cited as a COVID19 related barrier [32-34].

Although not unexpected, respondents reported high levels of distress while working from home. Compared to past Canadian data using the NCHA II distress items, a greater proportion of male and female respondents reported experiencing these symptoms of distress than reported on past surveys (S5 Table) [35]. Respondents are typically asked whether they experienced any of the NCHA II distress items over the past 12 month period. Our data displays a trend of more frequent instances of distress over a shorter period of time. This aligns the respondent reports of their own mental health and feelings of apathy being major barriers to getting work done at home. Unlike technological barriers, which for many could be solved with additional financial support to improve technology and internet connectivity, both negative mental health and loss of motivation do not have straightforward solutions. These cause long term impacts for people, even beyond the pandemic. There has been growing concern for lack of mental health services for graduate students and postdoctoral fellows [36,37]. The aftereffects of the pandemic may expedite this need for increased resources and professional support. Some respondents already reported seeking new or using previously established mental health supports to cope during this time. This, along with social connections with friends or family and emotional support from supervisors, paints 
a picture of respondents who are reaching out to a variety of sources to cope with the distress they are experiencing.

When looking at the key concerns of graduate students, many are pre-existing concerns that have been amplified by the pandemic, such as those related to research productivity and career prospects [37]. This is consistent with other postsecondary students across Canada, with recent data identifying that $67 \%$ were very or extremely concerned about their job prospects in the future [38]. Many institutions are already scaling back hiring of new academic research positions due to predicted economic uncertainty [39]. Due to an already small prospective job pool becoming even smaller as a result of these cuts, this has increased the pressure on graduate students and postdoctoral fellows to publish or perish. However, many at this time are unable to collect data due to laboratory closures, or are working at reduced capacity as laboratories slowly reopen. The pressure to perform in order to secure their financial and academic future has run up against the outside force of the pandemic, which they do not have control over. This lack of agency over when and how they will return to the laboratory can partially explain the high reported levels of distress, as well the concern that this will decrease their competitiveness as a researcher.

These concerns are also reflected in the support requested from supervisors; understanding and empathy surrounding the return to the laboratory and guidance on how to best approach the return to ensure productivity. As graduate students and postdoctoral fellows are already placing internal pressure and stress on themselves following laboratory shutdowns, they do not want to receive additional external pleasure from their supervisors. Instead, they want to receive emotional support from their mentor to process and cope with this stress. Respondents also want clear guidance and direction from their supervisors, thereby increasing productivity and data collection to minimize potential negative impacts respondents are predicting the laboratory closure will have on their career. Although both of these supports will be helpful to graduate students and postdoctoral fellows at large, the authors would also like to acknowledge they will be especially important for those researchers with children. Respondents with childcare responsibilities, primarily women, were a minority in the survey data, however, they had unique challenges trying to balance full-time research and parenting responsibilities. Data has already emerged showing women have begun to publish less and start fewer new projects during the pandemic, with increased caregiving responsibilities being cited as one potential explanation [40]. Supervisor understanding and guidance during this period will be key to minimize negative repercussions as a result of researchers with children decreasing research activities.

Other more generalizable COVID-19 related concerns reported by respondents mimic those amongst many Canadians: concerns about maintaining social connectedness, financial worries, concerns about the health of family, friends, or oneself $[41,42]$. Respondents reported lower levels of concern related to personal finances (56.6\%) than the total population of postsecondary students in Canada (77\%) [38]. This may be partially explained by respondents receiving stipends and other continued research funding, while the postsecondary population surveyed by Statistics Canada included undergraduate students who do not have these supports. Unlike many Canadians who are struggling to cover living expenses and food costs, we only had a handful of respondents cite these as major concerns [43]. This could be in part from 
differences between our target population and the Canadian population as a whole, as it has been documented that many individuals who are able to attend graduate school have pre-existing economic privileges $[44,45]$. In this sense, most financial concerns were tied to long term outcomes, such as not being able to find employment or provide for family in the future, rather than immediate concerns related to food or shelter. This is consistent with support requested from administration and universities and research institutions, asking for guarantees of long term financial and degree timeline support to minimize these longer- term concerns.

All of this is framed in the context of the emotional response to laboratory closures. Graduate students and postdoctoral fellows were grieving, in a variety of ways, the research that might have been and the loss of the pre-COVID-19 research landscape. Most respondents reported emotions associated with bargaining and depression stages of the Kübler-Ross grief construct, which can inform ways to approach supporting this population now and during the return to laboratories. Kübler-Ross described bargaining as "an attempt to postpone" an inevitable change from occurring [23]. Respondents are anxious, nervous, and afraid about how the laboratory closures will impact their research careers. By making these unconscious bargains with themselves, they are still trying to negotiate away for things to return to the way they were prior to COVD-19 $[23,46]$. With depression, respondents are realizing that the change they have been bargaining to avoid, is unavoidable [46]. Respondents in this stage are becoming aware that the consequences of missing multiple months of research time will impact them, leaving them discouraged. Both bargaining and depression stages have been documented in the literature as being associated with respondents beginning to come to terms with the new reality post-change $[23,46]$. This is a significant challenge, both cognitively and emotionally, and often results in respondents cycling between these two stages, as well as the anger stage [46]. This previously documented pattern is consistent with our data trends from respondents. What is known on how to support persons during these stages aligns with supports previously identified by respondents; listening to the person experiencing grief, and then providing empathy and compassion [46-48].

Coping and processing change events takes time. Our data suggests graduate students and postdoctoral fellows are still processing the impacts caused by COVID-19. For many junior researchers, the 2020 COVID-19 laboratory closures will be a turning point in their careers. In the coming months, the support provided by supervisors and administrators will be key in assisting graduate students and postdoctoral fellows to cope and adapt. It is important to note that supervisors and administrators may also be experiencing similar grief and stress responses to the COVID-19 pandemic. Further research into the shutdown experiences and support needed by those in more senior positions is necessary. As laboratories slowly begin to reopen, a new normal for research will be found. When this stability is established, this will allow for acceptance and hope to start growing again.

\section{Limitations}


One limitation of this study was the use of self-reported measures, which can be impacted by social desirability bias [49]. We attempted to minimize this by having an anonymous data gathering tool with options to skip questions to have respondents feel secure in giving their answers. Another limitation is our respondents' sample size is relatively small compared to the potential total number of graduate students and postdoctoral fellows in Canada. This small sample size could lead to population biases in experience, such as most of respondents being located in Ontario or Quebec and from biology or health research-based backgrounds. Additionally, the stress and increased responsibilities experienced by our target population may have led to potential respondents self- selecting to be those who have the time to participate in a qualitative research study. When applying our findings to their own unique contexts, readers should consult their graduate students and postdoctoral fellows to ensure the themes identified within this manuscript are reflective of their own experiences.

\section{Conclusion}

Overall, we have documented the experiences of graduate students and postdoctoral fellows conducting laboratory-based research during the COVID-19 research shutdown. We used the Kübler-Ross model as a lens to analyse respondents' grief and emotional response resulting from laboratory closures. We identified that unclear and inconsistent communication from supervisors and administrators led to a sense of chaos and confusion leading up to laboratory closures. The processes of stopping experiments, either with uniform or staggered closures, impacted respondents' emotional response to laboratory closures. Respondents reported experiencing high levels of distress while working from home. Barriers and supports to working at home were also identified. Concerns regarding the impact of laboratory closures on graduate students and postdoctoral fellows were identified, particularly those concerning competitiveness of candidate and availability of future research positions. We compiled a list of supports requested by graduate students and postdoctoral fellows to facilitate their return to the laboratory environment including personal protective equipment and protocols, understanding and empathy, guidance and direction, timeline support, and financial support.

\section{Declarations}

\section{Acknowledgements}

The authors would like to thank all individuals who helped share information about the study via social media and email. We would also like to thank all graduate students and postdoctoral fellows who gave their time to complete the survey and/or be interviewed. Without their participation with work would not have been possible. Thank you for letting us share your stories.

The authors state no conflict of interest. 


\section{References}

1. Servick K. Updated: Labs go quiet as researchers brace for long-term coronavirus Science. 2020. doi:10.1126/science.abb7259

2. Dalton R. Texas facilities count cost of tropical storm. Nature. 2001;411:

3. Dalton R. New Orleans researchers fight to salvage work from submerged labs. Nature. 2005;437: 300.

4. Rodriguez F, Selvaratnam R, Mann P, Kalariya R, Petersen JR. What's a Lab to Do During and After a Hurricane? Laboratory Medicine. 2018. pp. e23-e28. doi:1093/labmed/Imy006

5. Kohler CE, Gray WE. Earthquake Preparedness for Laboratories. Indiana University; Available: https://ehs.iu.edu/doc/lab-safety/earthquake-preparedness.pdf

6. Grimm D. "It"s heartbreaking.' Labs are euthanizing thousands of mice in response to coronavirus pandemic. Science. 2020. doi:1126/science.abb8633

7. Servick K, Cho A, Couzin-Frankel J, Guglielmi G. Coronavirus disruptions reverberate through research. Science. 2020;367: 1289-1290.

8. Arnold C. COVID-19: Biomedical research in a world under social-distancing Nature Medicine. 2020. doi:10.1038/d41591-020-00005-1

9. Bao W. COVID -19 and online teaching in higher education: A case study of Peking Human Behavior and Emerging Technologies. 2020. pp. 113-115. doi:10.1002/hbe2.191

10. Iyer P, Aziz K, Ojcius DM. Impact of COVID-19 on dental education in the United States. Journal of Dental Education. 2020. pp. 718-722. doi:1002/jdd.12163

11. Ashton P, Pintor-Escobar L. Five ways to tackle PhD research anxieties triggered by COVID-19 lockdowns. Nature. 2020. doi:1038/d41586-020-01898-1

12. Leshner Al. Restart science stronger after COVID-19. Science. 2020;369:

13. Ahmed MA, Arslan Ahmed M, Behbahani AH, Brückner A, Charpentier CJ, Morais LH, et The precarious position of postdocs during COVID-19. Science. 2020. pp. 957.2-958. doi:10.1126/science.abc5143

14. Johnson RL, Coleman RA, Batten NH, Hallsworth D, Spencer EE. The Quiet Crisis of PhDs and COVID19: Reaching the financial tipping point. doi:21203/rs.3.rs-36330/v2

15. Wang S. A student's guide to undergraduate research. Nature. 2019. doi:1038/d41586- 019-00871-x

16. Langin K. Amid concerns about grad student mental health, one university takes a novel Science. 2019. doi:10.1126/science.caredit.aay 7113

17. Being a PhD student shouldn't be bad for your health. Nature. 2019. pp. 307-307. doi:1038/d41586019-01492-0

18. Woolston C. A better future for graduate-student mental health. Nature. 2019. doi:1038/d41586-01902584-7 
19. American College Health Association. American College Health Association - National College Health Assessment II: Canadian Reference Group Data Report Spring 2016. 2016. Available: https://www.acha.org/documents/ncha/NCHAII\%20SPRING\%202016\%20CANADIAN\%20REFERENCE\%20GROUP\%20DATA\%20R pdf

20. Statistics Canada. Perceived life stress, 2014. Ottawa; 2015 Jun. Report No.: 2015001. Available: https://www150.statcan.gc.ca/n1/pub/82-625-x/2015001/article/14188-eng.htm

21. Moyser M. Gender differences in mental health during the COVID-19 pandemic. Statistics Canada; 2020 Jul. Available: https://www150.statcan.gc.ca/n1/pub/45-28- 0001/2020001/article/00047eng.htm

22. Mirowsky J, Ross CE. Sex Differences in Distress: Real or Artifact? American Sociological 1995. p. 449. doi:10.2307/2096424

23. Craytor JK, Kubler-Ross E. On Death and Dying. The American Journal of Nursing. p. 2710. doi:10.2307/3421124

24. Amundson NE, Borgen WA. The Dynamics of Unemployment: Job Loss and Job The Personnel and Guidance Journal. 1982. pp. 562-564. doi:10.1002/j.2164- 4918.1982.tb00723.x

25. A Look Through the Kubler-Ross Theoretical Lens. Theoretical Frameworks in Qualitative pp. 110128. doi:10.4135/9781412986335.n7

26. Elrod PD, David Elrod P, Tippett DD. The "death valley" of change. Journal of Organizational Change Management. 2002. pp. 273-291. doi:1108/09534810210429309

27. Malone E. The Kubler-Ross change curve and the flipped classroom: Moving students past the pit of despair. Education in the Health Professions. 2018. p. 36. doi:4103/ehp.ehp_26_18

28. Schoolfield M, Orduña A. Understanding staff nurse responses to change: utilization of a griefchange framework to facilitate innovation. Clin Nurse Spec. 1994;8: 57-62.

29. Kearney KS. Study of the Emotional Effects on Employees Who Remain Through Organizational Change: A View Through Kubler-ross (1969) in an Educational Institution. Hyle A, editor. PhD, Oklahoma State University. 2002.

30. Clapper AT. Loss, gain, and grief as a result of externally imposed demands for educational change (educational reform). Unpublished doctoral dissertation, Drake University.

31. Toronto Science Policy Network 2020. The Early Impacts of COVID-19 on Graduate Students across Canada. Available: https://toscipolicynet.wordpress.com/covid19-report/

32. Langin K. The pandemic is hitting scientist parents hard, and some solutions may Science. 2020. doi:10.1126/science.caredit.abe1220

33. Staniscuaski F, Reichert F, Werneck FP, de Oliveira L, Mello-Carpes PB, Soletti RC, et al. Impact of COVID-19 on academic mothers. Science. 2020;368:

34. Sallee M, Ward K, Wolf-Wendel L. Can Anyone Have it All? Gendered Views on Parenting and Academic Careers. Innovative Higher Education. 2015;41: 187-202. 
35. Linden B, Stuart H. Canadian Post-Secondary Student Mental Health and Wellbeing: A Descriptive Analysis. doi:31219/osf.io/4ajv6

36. Evans TM, Bira L, Gastelum JB, Weiss LT, Vanderford NL. Evidence for a mental health crisis in graduate education. Nat Biotechnol. 2018;36: 282-284.

37. Tsai JW, Muindi F. Towards sustaining a culture of mental health and wellness for trainees in the biosciences. Nat Biotechnol. 2016;34: 353-355.

38. Statistics Canada. Impacts of the COVID-19 pandemic on postsecondary students (ICPPS). 2020 May. Available: https://www150.statcan.gc.ca/n1/en/daily- quotidien/200512/dq200512a-eng.pdf? st=spyF2Ddb

39. Bodin M. University redundancies, furloughs and pay cuts might loom amid the pandemic, survey finds. Nature. 2020 [cited 6 Aug 2020]. doi:1038/d41586-020-02265-w

40. Viglione G. Are women publishing less during the pandemic? Here's what the data Nature. 2020. pp. 365-366. doi:10.1038/d41586-020-01294-9

41. LaRochelle-Côté S, Uppal S. Differences in the concerns of Canadians with respect to the COVID-19 pandemic. Statistics Canada; 2020 May. Available: https://www150.statcan.gc.ca/n1/en/pub/45-280001/2020001/article/00019-pdf?st=1HwKnGwq

42. LaRochelle-Côté S, Uppal S. The social and economic concerns of immigrants during the COVID-19 pandemic. Statistics Canada; 2020 May. Available: https://www150.statcan.gc.ca/n1/en/pub/45-280001/2020001/article/00012-pdf?st=0qIIZAXN

43. Daily Bread Food Bank. Hunger Lives Here: Risks and Challenges Faced by Food Bank Clients During Covid-19. 2020. Available: https://www.dailybread.ca/wp- content/uploads/2020/07/DB-COVIDImpact-Report-2020-Final-Web.pdf

44. Mullen AL, Goyette KA, Soares JA. Who Goes to Graduate School? Social and Academic Correlates of Educational Continuation after College. Sociology of Education. 2003. p. doi:10.2307/3090274

45. Lee EM. "'Where People Like Me Don't Belong"': Faculty Members from Low- socioeconomic-status Backgrounds. Sociology of Education. 2017. pp. 197-212. doi:1177/0038040717710495

46. Leybourne SA. Emotionally sustainable change: two frameworks to assist with International Journal of Strategic Change Management. 2016. p. 23. doi:10.1504/ijscm.2016.079633

47. Oates JR, Maani-Fogelman PA. Nursing Grief and Loss. StatPearls. Treasure Island (FL): StatPearls Publishing;

48. Shoolin JS. Change Management - Recommendations for Successful Electronic Medical Records Implementation. Appl Clin Inform. 2010;1:

49. Althubaiti A. Information bias in health research: definition, pitfalls, and adjustment $J$ Multidiscip Healthc. 2016;9: 211-217.

\section{Tables}


Table 1. Migration patterns of graduate students and postdoctoral fellows during the transition to working from home.

\begin{tabular}{l|c}
\hline Category & N (\%) \\
\hline Remained in same city as their laboratory & 224 \\
\hline $\begin{array}{l}\text { Traveled to a city within the same province or territory (Less than 1- } \\
\text { hour drive) }\end{array}$ & 26 \\
\hline $\begin{array}{l}\text { Traveled to a city within the same province or territory (More than } \\
\text { 1-hour drive) }\end{array}$ & $18.2)$ \\
\hline Traveled to another province or territory & $(6.4)$ \\
\hline Traveled to another country & $(4.6)$ \\
\hline
\end{tabular}

Table 2. Characteristics of working from home experiences by respondents across Canada. 


\begin{tabular}{|c|c|}
\hline Category & $\begin{array}{c}\mathbf{N} \\
(\%)\end{array}$ \\
\hline Dedicated Home Workspace & $52(20.4)$ \\
\hline No & $79(31.0)$ \\
\hline Yes, Shared Workspace & $124(48.6)$ \\
\hline Yes, Individual Workspace & \\
\hline $\begin{array}{c}\text { Participation in course(s) as part of planned studies prior to } \\
\text { shutdown }\end{array}$ & $183(71.5)$ \\
\hline No Yes, The course ended during shutdown & $63(21.9)$ \\
\hline Yes, The course transitioned online during shutdown & $(1.3)$ \\
\hline Yes, recently submitted or with an editor & $28(10.9)$ \\
\hline Project(s) in the peer review process & $164(64.1)$ \\
\hline No & $(6.3)$ \\
\hline Yes, Sent for peer review & $26(10.2)$ \\
\hline Yes, Completing revisions (major & 10 \\
\hline Yes, Submitted revisions & $(3.9)$ \\
\hline Yes, Recently accepted & 12 \\
\hline
\end{tabular}

Table 3. Opinions on household distribution of labour. Total population includes respondents who are non-binary or genderfluid, as well a those who did not disclose their gender. $\mathrm{N}$ values and percentage values are given for each row. Significance was determined by Fisher's exact test using GraphPad Prism 8. Significance was determined by Fisher's exact test using GraphPad Prism 8 ( $P=0.1100)$.

\begin{tabular}{l|c|c|c|}
\hline Category & $\begin{array}{c}\text { No - Uneven } \\
\text { distribution of labour }\end{array}$ & $\begin{array}{c}\text { Yes - Even } \\
\text { distribution of labour }\end{array}$ & $\begin{array}{c}\text { Total } \\
\text { Respondents }\end{array}$ \\
\hline $\begin{array}{l}\text { Female } \\
\text { Respondents }\end{array}$ & $53(36.8 \%)$ & $91(63.2 \%)$ & 144 \\
\hline $\begin{array}{l}\text { Male } \\
\text { Respondents }\end{array}$ & $11(23.4 \%)$ & $36(76.6 \%)$ & 47 \\
\hline $\begin{array}{l}\text { Total } \\
\text { Population }\end{array}$ & $75(33.9 \%)$ & $146(66.1 \%)$ & 221 \\
\hline
\end{tabular}


Table 4. Recommended Widespread Laboratory Shutdown Procedure Guidelines.

\section{During the Initial Shutdown Period}

- Provide clear communication regarding shutdown decisions to graduate students and postdoctoral fellows

Ensure communications and decisions are consistent between laboratories, departments, and research institutes at the same institution, where possible

If major differences in policy are required between research groups due to research contexts, make the reason shutdown procedures differ explicit to graduate students and postdoctoral fellows to minimize confusion

If possible, minimize sudden changes between policies that are contradictory over short time periods. In certain emerging situations, this may not be possible. In these cases, acknowledge why this decision has been made on short notice.

- Encourage uniform laboratory closures across departments and research institutions.

In the event staggered closures are necessary to preserve data, be explicit as to why some individuals are continuing to work when others are not. This will minimize the potential for an implied hierarchy between those with perceived 'high priority' and 'low priority' projects

- Set up remote access to laboratory servers and data for all lab members

Distribute technology from the laboratory (computers, monitors, hard drives, etc.) when possible for laboratory members to set up their homework spaces

Remind laboratory members to bring home more personal belongings and resources than they think they should (i.e. plan for a longer shutdown rather than a short one) 


\section{While working from home}

Encourage graduate students and postdoctoral fellows to develop and maintain a routine, as well as create a dedicated working space if possible

Help maintain routine and personal connections through virtual one on one meetings, laboratory meetings, journal clubs, and other social activities

When connecting with graduate students and postdoctoral fellows, inquire about their emotional and mental wellbeing in addition to progress on work

Provide information about mental health and financial supports available to students and fellows

Provide clear updates on changes in policy on returning to the laboratory or other relevant information

\section{Facilitating the return to the laboratory}

Continue to provide information about mental health and financial supports available to students and fellows

Be empathetic and provide moral support to students and fellows returning to the laboratory.

Be explicit in understanding the circumstances surrounding the return to the laboratory, including reduced levels of productivity and data production compared to pre- shutdown. Consult laboratory members on their circumstances and build flexibility into the return to work plans to account for individual differences (for example, researchers who are responsible childcare, are immunocompromised, international researchers, etc.) 
Have one on one meetings with returning graduate students and postdoctoral fellows to develop a plan to restart their project. Outline specific research goals and outcomes.

Ensure students and fellows will not be financially penalized for time lost during the laboratory shutdown, such as loss of funding for going overtime

Have explicit guidelines as to how degree milestones and timelines will be affected by the laboratory shutdown. If changes are made to previously set guidelines before the shutdown, make these changes in consultation with students who will be affected.

\section{Figures}

A.
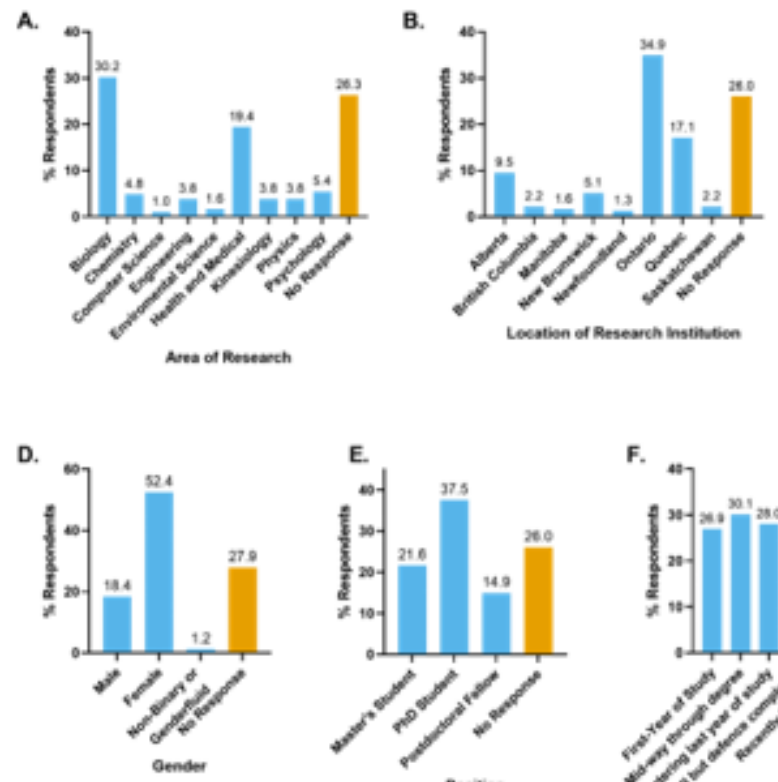

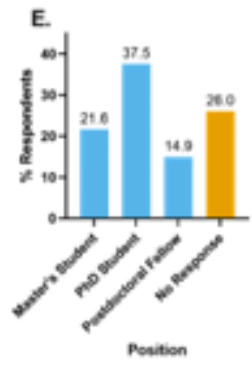

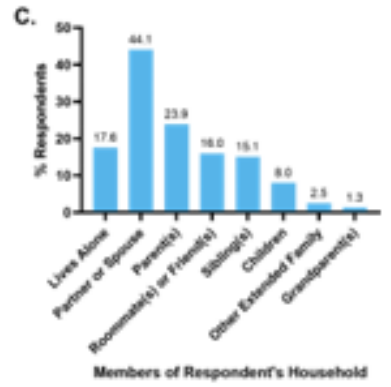

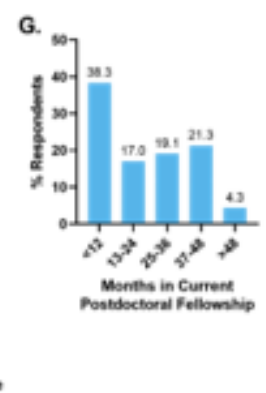

\section{Figure 1}

Survey Respondent Characteristics. A. Respondent area of research, N=315. B. Location of Respondent's research institution, $N=315$. C. Living situation of the respondent while working from home. Percentage of respondents with listed members of the household is shown. $N=238$. D. Gender of respondents, N=315.E. Academic position of respondents, $\mathrm{N}=315$. F. Progress in graduate degrees for Master's and PhD students. $\mathrm{N}=186$. $\mathrm{G}$. Months in postdoctoral fellowship position, $\mathrm{N}=47$. 
A.

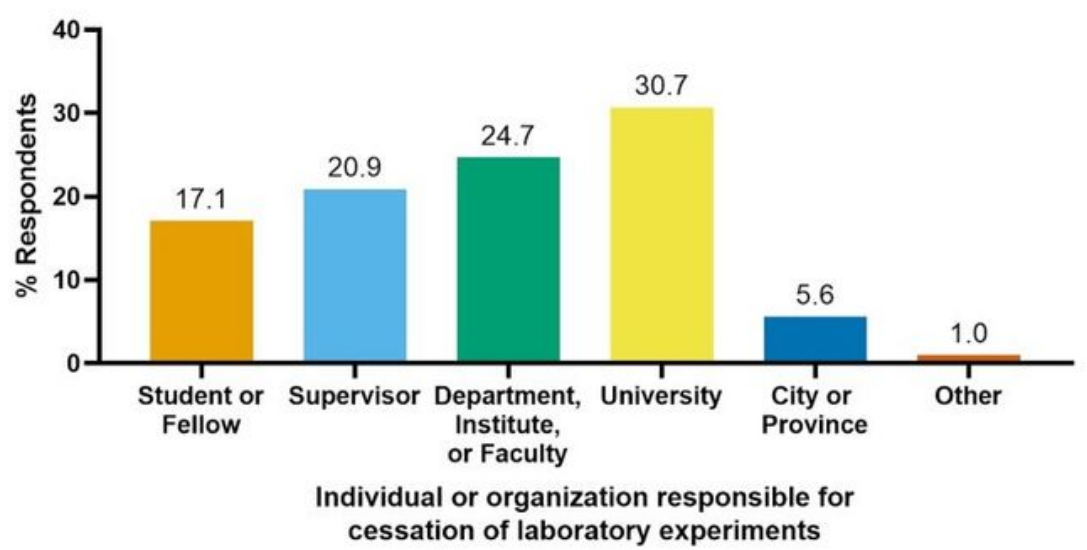

C.

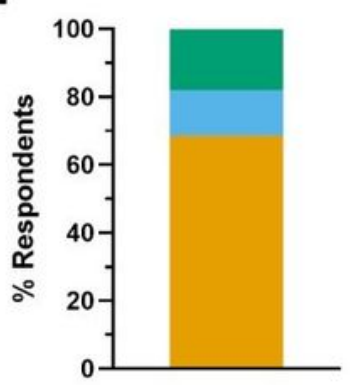

SOP made in response to COVID-19

SOP made prior to COVID-19

No SOP for Laboratory Shutdown
B.

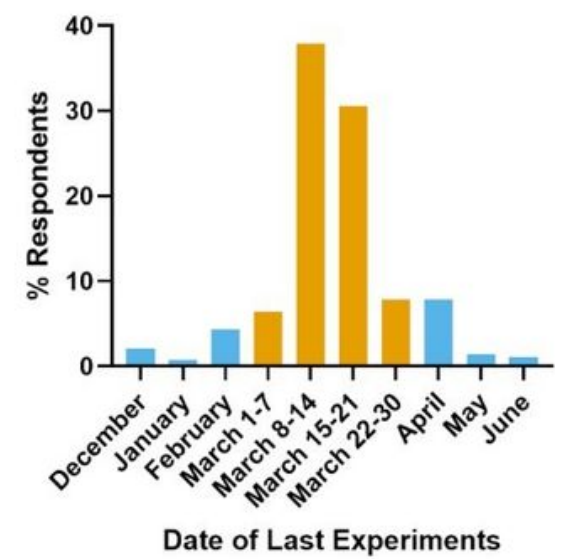

E.

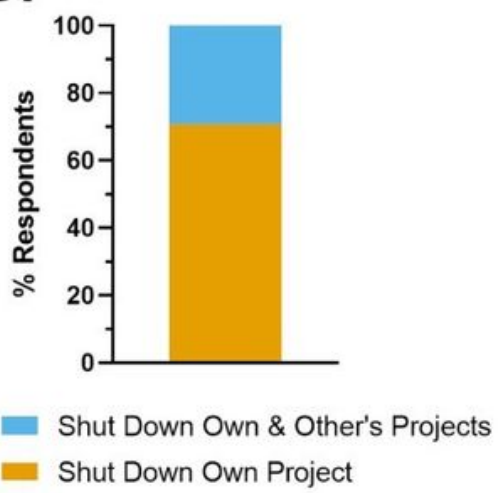

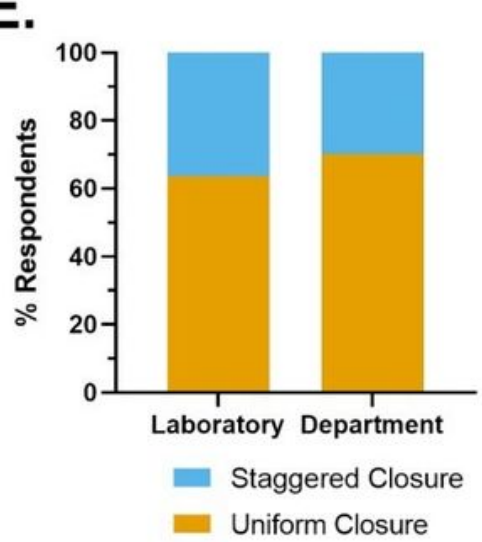

$\mathbf{F}$

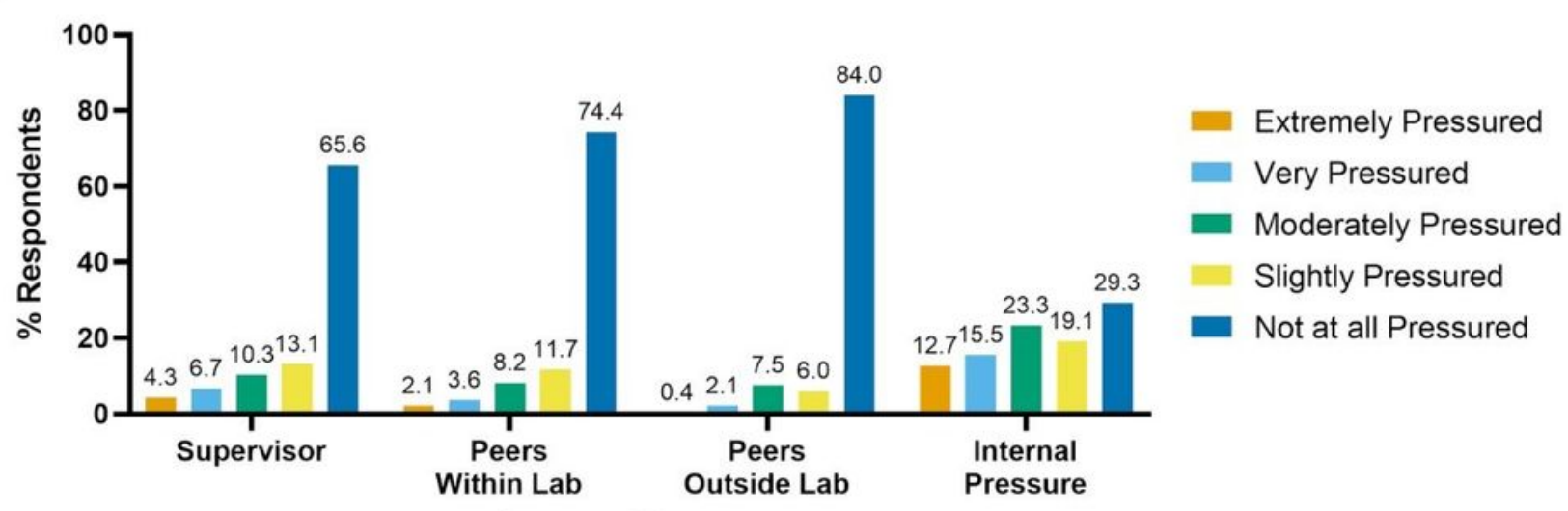

Source of Pressure

Figure 2

Characteristics of laboratory shutdowns experienced by respondents across Canada. A. Respondent description of the individual or organization, including the respondent themselves, which made the decision to cease laboratory experience. B. Date of last experiments performed by respondents. C. Prevalence of Standard Operating Procedures (SOPs) for fast laboratory closures. D. Project shutdown responsibilities of respondents. E. Closure practices of respondent laboratories and departments. Uniform 
closure describes scenarios where all work stopped at approximately the same time. Staggered closure describes scenarios where some individuals transitioned to work from home while others continued to work in lab. F. Prevalence of pressure to keep working during laboratory closures.

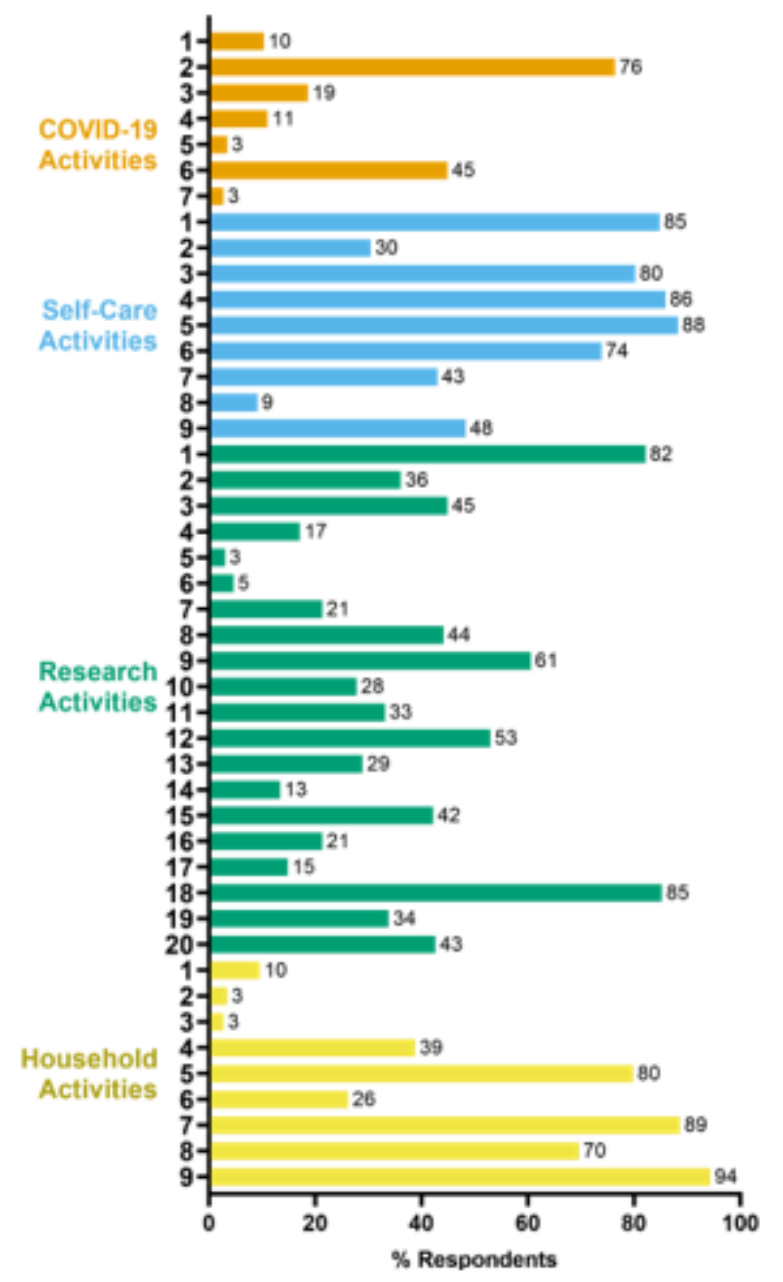

\section{Figure 3}

Activities conducted by graduate students and postdoctoral fellows while working from home. Values are displayed in percentage of total respondents across COVID-19, Self-Care, Research, and Household related activities. $\mathrm{N}=263$. Full list of activity descriptions available in supplementary table 1. 


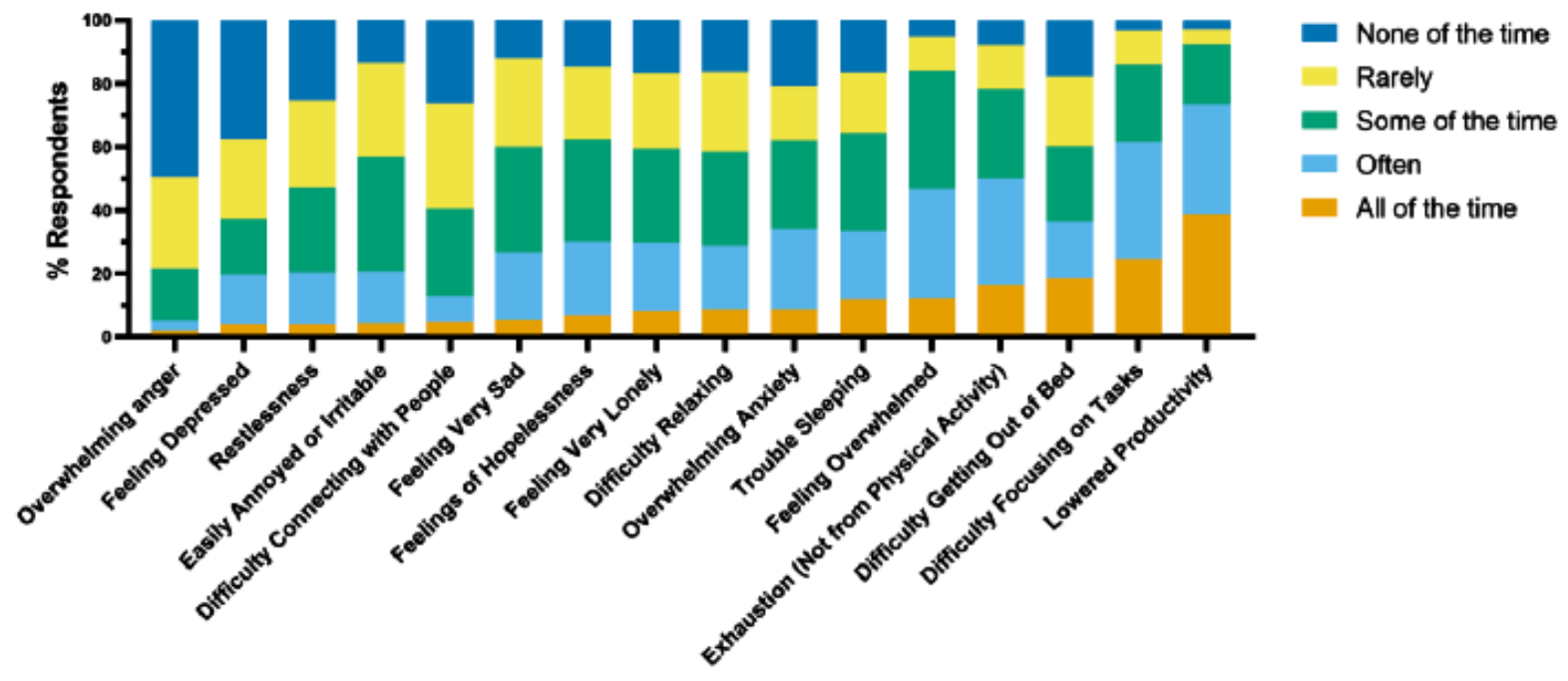

Figure 4

Self-reported symptoms of distress while working from home during COVID-19. Values are displayed in percentage of total respondents across 16 symptoms of distress. $\mathrm{N}=254-256$. Items are ranked from lowest to highest percentage of respondents reporting feeling "All of the time".

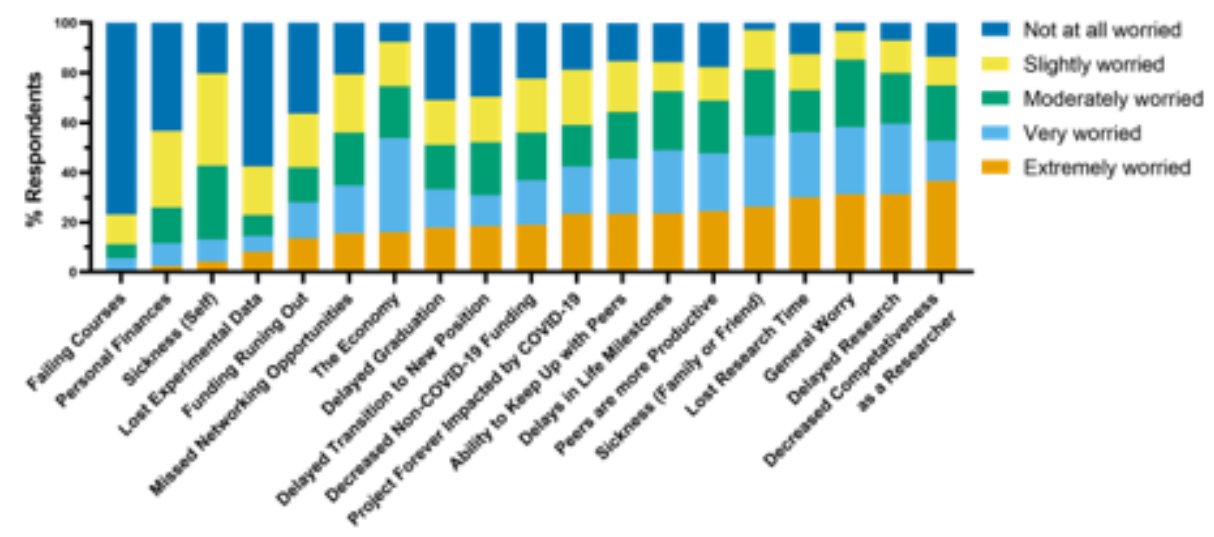

Figure 5

Graduate students and postdoctoral fellows' concerns related to the COVID-19 pandemic. Values are displayed in percentage of total respondents across 19 potential areas of concern. $\mathrm{N}=234-237$. Items are ranked from lowest to highest percentage of respondents reporting feeling "all of the time". 
A.

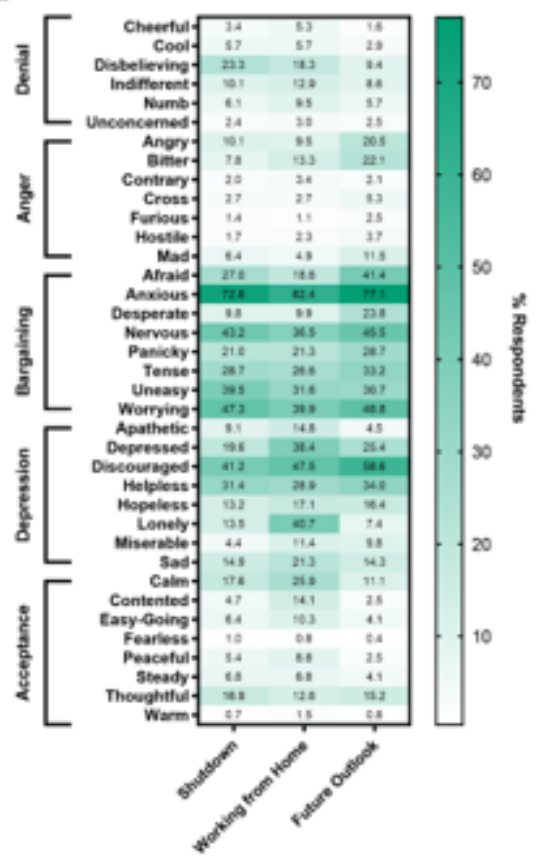

B.

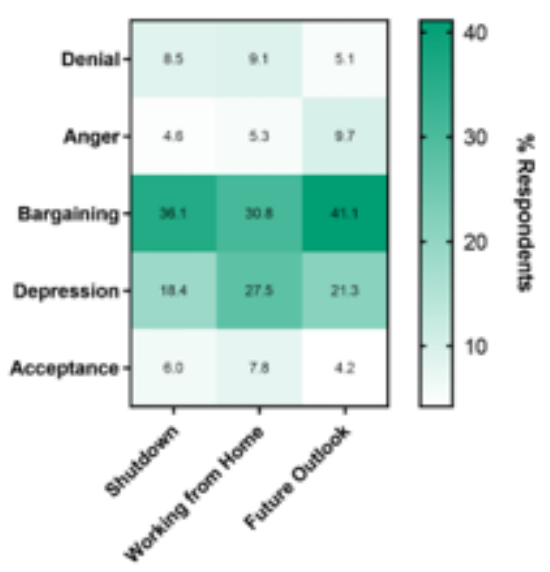

\section{Figure 6}

Kübler-Ross analysis of emotions experienced by graduate students and postdoctoral fellows across timepoints. Percentage of respondents reporting indicated emotions during laboratory shutdown, while working from home, and when they think of the future. Categorization of emotions by the Kübler-Ross model of grief based on work by Clapper (1991). A. Full list of emotions experienced during COVID-19. B. Composite score of overall Kübler- Ross groupings of emotions

\section{Supplementary Files}

This is a list of supplementary files associated with this preprint. Click to download.

- Suartetal.2020COVID19SurveySupportingMaterial.pdf 